\title{
Synthesis of 2-(substituted-phenyl)-5-(aminomethyl)- and (thiomethyl)-1,3,4-oxadiazoles. Oxidation of thiomethyl-oxadiazole derivatives by dimethyldioxirane
}

\author{
Attila Kiss-Szikszai ${ }^{a}$, Tamás Patonay*a and József Jekő ${ }^{b}$ \\ ${ }^{a}$ Department of Organic Chemistry, University of Debrecen, P.O.Box 20, H-4010 Debrecen, \\ Hungary \\ ${ }^{b}$ ICN Hungary Co. Ltd., H-4440 Tiszavasvári, Hungary \\ E-mail:tpatonay@tigris.klte.hu
}

Dedicated to Prof. Kalevi Pihlaja on the occasion of his $60^{\text {th }}$ birthday

(received 03 Apr 01; accepted 01 Jan 99; published on the web 15 Apr 02)

\begin{abstract}
Various substituted 2-aryl-5-aminomethyl- and -5-thiomethyl-1,3,4-oxadiazoles were synthesized in high yields from the corresponding chloromethyl derivatives. Selective oxidation of the sulfides into 5-sulfinylmethyl and 5-sulfonylmethyl-1,3,4-oxadiazoles by dimethyldioxirane under mild conditions was also demonstrated.
\end{abstract}

Keywords: Dimethyldioxirane, nucleophilic substitution, 1,3,4-oxadiazole

\section{Introduction}

Derivatives of 1,3,4-oxadiazole constitute an important family of heterocyclic compounds. ${ }^{1}$ Since many of them display a remarkable biological activity, their synthesis and transformations have been received particular interest for a long time. The 2-aryl-5-(substituted methyl)-1,3,4oxadiazoles have been reported to show antibacterial, ${ }^{2,3}$ antifungal, ${ }^{4}$ analgesic and antiinflammatory, ${ }^{5,6}$ and hypoglycemic ${ }^{3}$ activity. Their synthetic usefulness has also been demonstrated. The 2-azidomethyl-5-(4-chlorophenyl)-1,3,4-oxadiazole, as a 1,3-dipole, added efficiently to norbornene derivatives ${ }^{7}$ while 2-phenyl-5-[(2-pyridylium)methyl]-1,3,4-oxadiazole chloride was found to be a good nitrone precursor $^{8}$. Liebscher and his coworkers reported diastereoselective side-chain alkylation of oxadiazoles by using a prolinolyl group as a chiral auxiliary ${ }^{9}$ and also the synthesis of bicyclic imidazoles carrying oxadiazolyl moiety. ${ }^{10}$ To prepare the corresponding substrates, nucleophilic substitution of 2-aryl-5-chloromethyl-1,3,4oxadiazoles have usually been applied.

The most popular synthesis of 2,5-disubstituted-1,3,4-oxadiazole based on the thermal or 
acid-catalysed cyclization of 1,2-diacylhydrazines ${ }^{1}$. Ring-closure usually proceeded in the presence of hot phosphorus oxychloride ${ }^{3,411}$ although an improved method by using triphenylphospine/carbon tetrachloride/triethylamine reagent was also reported recently. ${ }^{2}$ Oxidative cyclization of aldehyde or ketone acylhydrazones ${ }^{1}$ and thermal acylation of 5substituted tetrazoles followed by nitrogen elimination ${ }^{12-14}$ also afford the desired derivatives, but these approaches have not been used for the synthesis of 2-aryl-5-chloromethyl-1,3,4oxadiazoles.

Recently we have studied the preparation of 2-(alkylamino- or dialkylaminomethyl]- and 2hetarylthiomethyl-5-aryl-1,3,4-oxadiazoles and selected results of this project are presented in this contribution.

\section{Results and Discussion}

The starting materials 1-3 were synthesized from the corresponding aroyl hydrazides and chloroacetyl chloride according to a reported procedure ${ }^{4,15}$. From the various conditions of their nucleophilic substitution reported in the literature (2 equiv. of nucleophile in dioxane, ${ }^{5,6} 1-1.2$ equiv. of nucleophile in methanol or ethanol solution in the presence of potassium carbonate ${ }^{3}$, sodium acetate 4 or triethyl amine, ${ }^{9} 1$ equiv. of nucleophile and 1 equiv. of sodium hydride in $\mathrm{DMF}^{10}, 1$ equiv. of nucleophile and potassium carbonate in DMSO solution ${ }^{15}$ ), the last one was found to give the highest yields. The reaction of chloromethyl derivatives $\mathbf{1 , 2}$ with various amines or hetarenethiols and potassium carbonate in DMSO solution at room temperature gave the expected amines 4,5 and sulfides 6,7 in good yields in 4-6 hrs (Scheme 1). Surprisingly, no reaction was observed upon analogous treatment of 2-chloromethyl-5-(3,4,5-trimethoxyphenyl)1,3,4-oxadiazole (3) even at a longer reaction period. At higher temperatures the starting material was consumed but the expected product was not obtained. It is very likely that under these conditions the concurrent attack of the nucleophile(s) on the C-2, C-5 carbon atoms of the oxadiazole ring takes place which leads to ring cleavage. ${ }^{1}$

When sulfides $\mathbf{6 b}, \mathbf{7 b}$ were treated with dimethyldioxirane ${ }^{16}$ (DMD), the corresponding sulfoxides $\mathbf{8 b}, 9 \mathbf{b}$ and sulfones $\mathbf{1 0 b}, \mathbf{1 1 b}$ were obtained in excellent yields depending on the amount of oxidizing agent used. Treatment of the sulfides with 2-4 equiv. of DMD resulted in the formation of sulfoxides, the synthesis of sulfones required a higher amount (5-8 equiv.) of oxidizing agent. The oxadiazole moiety remained intact (Scheme 1). The measured sulfoxide/sulfone ratio of the crude product in the synthesis of sulfoxides was higher than 9/1 which indicates a good chemoselectivity for the attack of DMD on the sulfur atom of the sulfide unit. This reactivity pattern is characteristic of DMD oxidation although exceptional deviations have also been reported. ${ }^{17}$ It is noteworthy that a complete decomposition of the starting $\mathbf{6 , 7}$ sulfides without the formation of considerable amount of any sulfoxide or sulfone was found in the attempted oxidation by hydrogen peroxide in acetic acid. Once again, these results demonstrate the synthetic value of DMD in oxygen transfer under mild and neutral conditions. 


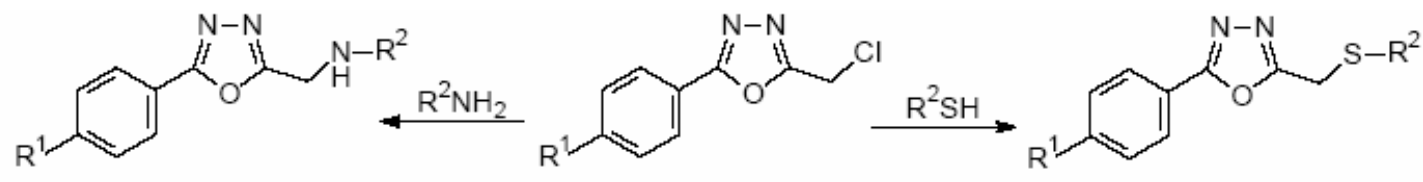

$4 a-f, 5 a-d$

6a-d, $7 a, b, e$

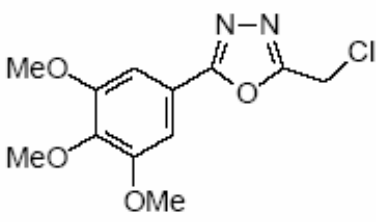

3

1, 4, 6, 8, $10 \quad \mathrm{R}^{1}=\mathrm{Cl}$

2, 5, 7, 9, $11 \quad R^{1}=$ OMe
1,2

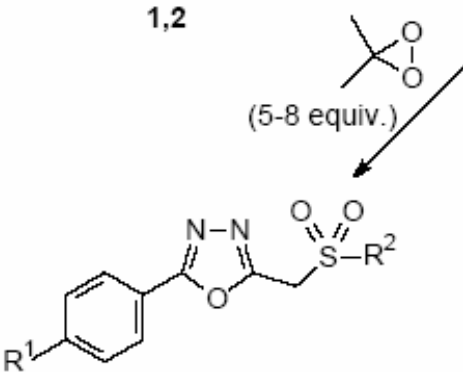

$10 \mathrm{~b}, 11 \mathrm{~b}$

$$
\begin{aligned}
\text { 6-11 a } & \mathrm{R}^{2}=2 \text {-pyridyl } \\
\text { b } & \mathrm{R}^{2}=2 \text {-benzimidazolyl } \\
\text { c } & \mathrm{R}^{2}=2 \text {-benzoxazolyl } \\
\text { d } & \mathrm{R}^{2}=1,2,4 \text {-triazol-3-yl } \\
\text { e } & \mathrm{R}^{2}=2 \text {-benzothiazolyl }
\end{aligned}
$$

$8 b, 9 b$

\section{Scheme 1}

The structures of the obtained products were proven by spectroscopic methods. The characteristic $1 \mathrm{C}=\mathrm{N}$ band (1616-1594 cm-1) of medium intensity and a medium-strong band at 1025-1000 cm-1 were identified in each IR spectra, the latter could be attributed to the C-O-C vibration or heteroatom ring deformation of the oxadiazole ring. ${ }^{18}$ The presence of the $1,3,4$ oxadiazole unit was supported by the appearance of two quaternary signals $(\mathrm{C}-2, \mathrm{C}-5)$ in the range $\delta=162.5-166.6 \mathrm{ppm}$ of their ${ }^{13} \mathrm{C}$ NMR spectrum. The oxadiazole ring was found to exert a slight upfield shift (ca. $-5 \mathrm{ppm}$ ) on the ipso-carbon of the 2-aryl group. In their MS spectra 5chloromethyl derivatives 1-3 and 5-thiomethyl derivatives 6,7 gave molecular ions of medium intensity and the base peak usually belonged to the corresponding acylium ions and nitrile radical ions which formed by the cleavage of the heteroatom ring. Both of these have been reported as characteristic 1 for this family. However, only very weak molecular ion were detected in the spectra of the aminomethyl derivatives 4,5 as the ArCO fragment appeared as a peak of medium intensity and the base peak usually came from the amine side chain. The most important fragmentation pathways are shown by Scheme 2 . 


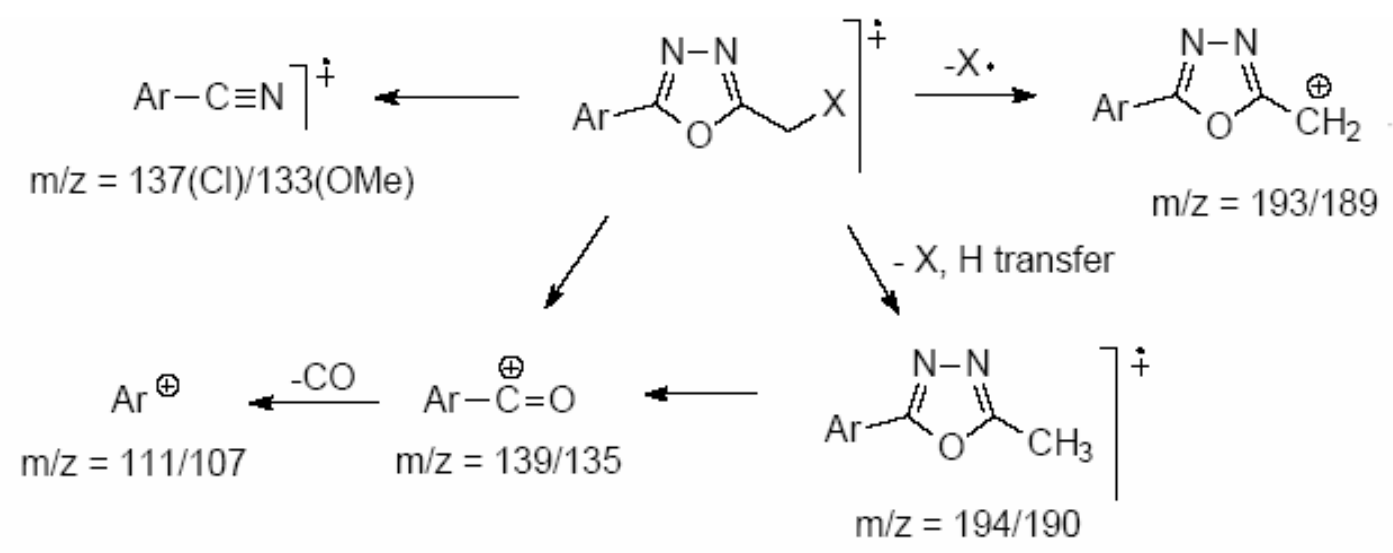

Scheme 2

\section{Experimental Section}

General Procedures. Dimethyldioxirane solution was prepared according to literature procedure $^{19}$ and its peroxide content was determined iodometrically. Chromatographic separations were performed using silica gel (Merck, 70-230 mesh). Thin-layer chromatography was carried out on Macherey-Nagel precoated silica plate $(0.25 \mathrm{~mm}$ layer thickness $)$. Melting points were determined on a Boetius hot-stage apparatus and are uncorrected. ${ }^{1} \mathrm{H} \mathrm{NMR}$ and ${ }^{13} \mathrm{C}$ NMR spectra were recorded with a Gemini 200 spectrometer in $\mathrm{CDCl}_{3}$ solution unless otherwise specified (internal standard TMS, $\delta=0 \mathrm{ppm}$ ). Mass spectra were taken on a VG Trio-2 (EI, $70 \mathrm{eV}$ ) apparatus. IR spectra were recorded with a Perkin-Elmer 283 instrument in $\mathrm{KBr}$ disks.

2-Aryl-5-(chloromethyl)-1,3,4-oxadiazoles 1, 2, 3 were prepared by a direct reaction of aromatic acidhydrazides and $\mathrm{ClCH}_{2} \mathrm{COCl}$ according to the procedure of Vakula et al.. ${ }^{15}$

5-Chloromethyl-2-(4-chlorophenyl)-1,3,4-oxadiazole (1). Colourless needles, yield 67\%, mp 80.5-81 ${ }^{\circ} \mathrm{C}$ (PhMe-hexane) (lit. $\left.{ }^{11} 85^{\circ} \mathrm{C}\right) . \mathrm{IR}: 3012,2960,1604(\mathrm{C}=\mathrm{N}), 1481,1410,1255,1092$ (Ar-Cl), 1011, 835, 735, $727 \mathrm{~cm}^{-1} .{ }^{1} \mathrm{H}$ NMR (DMSO-d $): 5.15\left(\mathrm{~s}, 2 \mathrm{H}, \mathrm{CH}_{2}\right), 7.70$ (d, J = 8.7Hz, 2H, 3',5'-H), 8.05 (d, $\left.J=8.7,2 \mathrm{H}, 2^{\prime}, 66^{\prime}-\mathrm{H}\right) .{ }^{13} \mathrm{C}$ NMR (DMSO-d $): 33.1\left(\mathrm{CH}_{2}\right), 121.9\left(\mathrm{C}-1^{\prime}\right), 128.7$ (C-2',6'), 129.9 (C-3',5'), 137.4 (C-4'), 163.3, 164.5 (C-2, C-5). MS: 228/230/232 (35/21/5, M', $\left.\mathrm{Cl}_{35} / \mathrm{Cl}_{37} / 2 \mathrm{xCl}_{37}\right), 193(28, \mathrm{M}-\mathrm{Cl}), 179\left(10, \mathrm{M}-\mathrm{CH}_{2} \mathrm{Cl}\right), 139\left(100,4-\mathrm{ClC}_{6} \mathrm{H}_{4} \mathrm{CO}^{+}\right), 137$ (29, 4$\left.\mathrm{ClC}_{6} \mathrm{H}_{4} \mathrm{CN}\right), 123$ (16), $111\left(50,4-\mathrm{ClC}_{6} \mathrm{H}_{4}{ }^{+}\right), 75(53)$.

5-Chloromethyl-2-(4-methoxyphenyl)-1,3,4-oxadiazole (2). Colourless prisms, yield 61\%, mp 88.5-90 ${ }^{\circ} \mathrm{C}$ (PhMe-hexane) (lit. ${ }^{11} 94-95{ }^{\circ} \mathrm{C}$, lit.19 $\left.92{ }^{\circ} \mathrm{C}\right)$. IR: 3018, $1616(\mathrm{C}=\mathrm{N}), 1497,1428$, 1310, 1259 (C-O-C), 1178, 1008, 843, 744, $737 \mathrm{~cm}^{-1} .{ }^{1} \mathrm{H}$ NMR (DMSO-d 6 ): 3.87 (s, 3H, MeO), $5.12\left(\mathrm{~s}, 2 \mathrm{H}, \mathrm{CH}_{2}\right), 7.15\left(\mathrm{~d}, J=9.0 \mathrm{~Hz}, 2 \mathrm{H}, 3^{\prime}, 5^{\prime}-\mathrm{H}\right), 7.95\left(\mathrm{~d}, J=9.0 \mathrm{~Hz}, 2 \mathrm{H}, 2^{\prime}, 6^{\prime}-\mathrm{H}\right) .{ }^{13} \mathrm{C}$ NMR $\left(\mathrm{DMSO}_{\mathrm{d}}\right)$ ): $33.2\left(\mathrm{CH}_{2}\right), 55.5(\mathrm{MeO}), 115.2\left(\mathrm{C}-3^{\prime}, 5^{\prime}\right), 115.3\left(\mathrm{C}-1^{\prime}\right), 128.8\left(\mathrm{C}-2^{\prime}, 6^{\prime}\right), 162.6,165.3$ (C-2, C-5, C-4'). MS: 224/226 (29/9, $\left.\mathrm{M}^{+}, \mathrm{Cl}_{35} / \mathrm{Cl}_{37}\right), 189$ (20, M - Cl), 135 (100, 4- 
$\mathrm{MeOC}_{6} \mathrm{H}_{4} \mathrm{CO}^{+}$), $133\left(20,4-\mathrm{MeOC}_{6} \mathrm{H}_{4} \mathrm{CN}\right), 119$ (8), 92 (15), 77 (23).

5-Chloromethyl-2-(3,4,5-trimethoxyphenyl)-1,3,4-oxadiazole (3). Colourless needles, yield 68\%, mp 106.5-110 ${ }^{\circ} \mathrm{C}$ (PhMe-hexane) (lit. $\left.{ }^{15} 215{ }^{\circ} \mathrm{C}\right)$. IR: 3008, $1594(\mathrm{C}=\mathrm{N}), 1498,1462,1418$, 1322, 1240 (C-O-C, OMe), 1130 (C-O-C, OMe), 996, 846, 744, $728 \mathrm{~cm}^{-1}$. ${ }^{1} \mathrm{H}$ NMR: 3.92 (s, 3H, 4-OMe), 3.96 (s, 6H, 3,5-OMe), 4.80 (s, 2H, $\left.\mathrm{CH}_{2}\right), 7.90$ (s, 2H, 2,6-H). ${ }^{13} \mathrm{C}$ NMR: $32.9\left(\mathrm{CH}_{2}\right)$, 56.3 (3',5'-MeO), 60.9 (4'-MeO), 104.3 (C-2',6'), 118.2 (C-1'), 141.4 (C-4'), 153.6 (C-3',5'), 162.0, 165.8 (C-2, C-5). MS: 284/286 (48/16, M+ $\left.{ }^{+}, \mathrm{Cl}_{35} / \mathrm{Cl}_{37}\right), 269$ (20, M-Me), 249 (4, M-Cl), $195\left(100,(\mathrm{MeO}){ }_{3} \mathrm{C}_{6} \mathrm{H}_{2} \mathrm{CO}^{+}\right)$.

2-(4-(Chlorophenyl)-5-(tert-butylamino)methyl-1,3,4-oxadiazole (4a). Typical procedure. $1.37 \mathrm{~mL}(13.037 \mathrm{mmol})$ of tert-butylamine and $1.86 \mathrm{~g}(13.5 \mathrm{mmol})$ of anhydrous $\mathrm{K}_{2} \mathrm{CO}_{3}$ were added to a stirred solution of $1(1.498 \mathrm{~g}, 6.540 \mathrm{mmol})$ in DMSO (30 mL) at room temperature and the reaction was monitored by TLC $(\mathrm{MeOH}-\mathrm{PhMe}=10: 1, \mathrm{v} / \mathrm{v})$. After 4 hours it was poured into water $(200 \mathrm{~mL})$. The precipitate was filtered off and washed with hexane $(2 \times 10 \mathrm{~mL})$ to obtain $1.165 \mathrm{~g}(67 \%)$ of $\mathbf{4 a}$ as white microcrystalline powder. $\mathrm{mp}$ 72.5-73.5 ${ }^{\circ} \mathrm{C}$ (EtOAc-hexane). IR: $3339(\mathrm{NH}), 2967\left(\mathrm{CH}_{3}\right), 1607(\mathrm{C}=\mathrm{N})$, 1584, 1482, 1409, 1364, $1091(\mathrm{Ar}-\mathrm{Cl}), 1010,844,729$ $\mathrm{cm}^{-1} .{ }^{1} \mathrm{H}$ NMR: 1.19 (s, 9H, tBu), 1.51 (s, 1H, NH), $4.10\left(\mathrm{~s}, 2 \mathrm{H}, \mathrm{CH}_{2}\right), 7.46$ (d, J = 6.8 Hz, 2H, 3',5'-H), 8.00 (d, $\left.J=6.8 \mathrm{~Hz}, 2 \mathrm{H}, 2^{\prime}, 66^{\prime}-\mathrm{H}\right) .{ }^{13} \mathrm{C} \mathrm{NMR:} 28.7\left(\mathrm{CMe}_{3}\right), 37.8\left(\mathrm{CH}_{2}\right), 51.0\left(\mathbf{C M e}_{3}\right)$, 122.5 (C-1'), 128.3 (C-2',6'), 129.5 (C-3',5'), 138.1 (C-4'), 164.4, 166.6 (C-2, C-5). MS: 250 (54, $\left.\mathrm{M}-\mathrm{CH}_{3}\right), 193$ (5, M - tBuNH), 181 (12), 139 (29, 4- $\left.\mathrm{ClC}_{6} \mathrm{H}_{4} \mathrm{CO}^{+}\right), 111\left(15,4-\mathrm{ClC}_{6} \mathrm{H}_{4}^{+}\right), 95$ (20), 70 (100, $\left.\mathrm{C}_{4} \mathrm{H}_{8} \mathrm{~N}\right)$. Anal. Calcd. for $\mathrm{C}_{13} \mathrm{H}_{16} \mathrm{ClN}_{3} \mathrm{O}$ (265.74): C, 58.76; H, 6.07; N, 15.81. Found: C, 58.77; H, 5.89; N, 15.44 .

5-(Benzylamino)methyl-2-(4-chlorophenyl)-1,3,4-oxadiazole (4b). From chloride 1 $(1.541 \mathrm{~g}, 6.727 \mathrm{mmol})$ and benzylamine $(1.48 \mathrm{~mL}, 13.549 \mathrm{mmol})$ as given for 4a. Yield 86\%, mp 69.5-71 ${ }^{\circ} \mathrm{C}$. IR: $3372(\mathrm{NH}), 2923,1606(\mathrm{C}=\mathrm{N}), 1484,1453,1412,1412,1133,1097,(\mathrm{Ar}-\mathrm{Cl})$, 1016, 830, 742, $736 \mathrm{~cm}^{-1} .{ }^{1} \mathrm{H}$ NMR: 1.90 (s, 1H, NH), 3.90 (s, 2H, PhCH $), 4.10$ (s, 2H, $\left.\mathrm{HetCH}_{2}\right), 7.25-7.36(\mathrm{~m}, 5 \mathrm{H}, \mathrm{Ph}), 7.50\left(\mathrm{~d}, J=6.9 \mathrm{~Hz}, 2 \mathrm{H}, 3^{\prime}, 5^{\prime}-\mathrm{H}\right), 7.96$ (d, $J=6.9 \mathrm{~Hz}, 2 \mathrm{H}, 2^{\prime}, 6^{\prime}-$ H). ${ }^{13} \mathrm{C}$ NMR: $42.8\left(\mathrm{HetCH}_{2}\right), 52.9\left(\mathrm{PhCH}_{2}\right), 122.4\left(\mathrm{C}-1^{\prime}\right), 127.5(\mathrm{C}-4 "), 128.3,128.4,128.7$, 129.6 (C-2',6', C-3',5', C-2",6", C-3",5"), 138.2, 139.1 (C-4', C-1"), 164.5, 165.6 (C-2, C-5). MS: $298(<1, \mathrm{M}-1), 194(20, \mathrm{M}-\mathrm{PhCH}=\mathrm{NH}), 139\left(10,4-\mathrm{ClC}_{6} \mathrm{H}_{4} \mathrm{CO}^{+}\right), 137\left(8,4-\mathrm{ClC}_{6} \mathrm{H}_{4} \mathrm{CN}\right), 106$ $\left(100, \mathrm{PhCH}_{2} \mathrm{NH}^{+}\right), 91\left(41, \mathrm{PhCH}_{2}{ }^{+}\right)$. Anal. Calcd. for $\mathrm{C}_{16} \mathrm{H}_{14} \mathrm{ClN}_{3} \mathrm{O}$ (299.75): C, 64.11; H, 4.71; N, 14.02. Found: C, 63.94; H, 4.58; N, 14.22.

2-(4-Chlorophenyl)-5-(cyclohexylamino)methyl-1,3,4-oxadiazole (4c). From chloride 1 $(1.513 \mathrm{~g}, 6.605 \mathrm{mmol})$ and cyclohexylamine $(1.52 \mathrm{~mL}, 13.287 \mathrm{mmol})$ as given for 4a. Yield 94\%, mp 82.5-85 ${ }^{\circ} \mathrm{C}$. IR: $3310(\mathrm{NH}), 2930\left(\mathrm{CH}_{2}\right)$, 2853, 1584, 1483, 1413, 1203, 1133, 1095 (Ar-Cl), 1011, 855, 834, $732 \mathrm{~cm}^{-1} .{ }^{1} \mathrm{H}$ NMR: 1.20 (m, 4H, cyclohexyl $\left.\mathrm{CH}_{2}\right), 1.70(\mathrm{~m}, 4 \mathrm{H}$, cyclohexyl $\left.\mathrm{CH}_{2}\right), 1.92\left(\mathrm{~m}, 2 \mathrm{H}\right.$, cyclohexyl $\left.\mathrm{CH}_{2}\right), 3.56(\mathrm{~m}, 1 \mathrm{H}$, cyclohexyl $\mathrm{CH}), 4.12(\mathrm{~s}, 2 \mathrm{H}$, $\left.\mathrm{HetCH}_{2}\right), 7.50$ (d, $\left.J=8.7 \mathrm{~Hz}, 2 \mathrm{H}, 3^{\prime}, 5^{\prime}-\mathrm{H}\right), 8.00$ (d, $\left.J=8.7 \mathrm{~Hz}, 2 \mathrm{H}, 2^{\prime}, 66^{\prime}-\mathrm{H}\right) .{ }^{13} \mathrm{C}$ NMR: 24.6 (C3",5"), 25.8 (C-4"), 33.0 (C-2',6"), 40.9 (HetCH2), 55.9 (C-1"), 122.5 (C-1'), 128.3, 129.6 (C2',6', C-3',5'), 138.1 (C-4'), 164.5, 166.1 (C-2, C-5). MS: $291\left(<1, \mathrm{M}^{+}\right), 290(<1, \mathrm{M}-1), 248$ (10, $\left.\mathrm{M}-\mathrm{C}_{3} \mathrm{H}_{7}\right), 194$ (12), $139\left(13,4-\mathrm{ClC}_{6} \mathrm{H}_{4} \mathrm{CO}^{+}\right), 111\left(6,4-\mathrm{ClC}_{6} \mathrm{H}_{4}^{+}\right), 98$ (100, $\left.\mathrm{cHxNH}^{+}\right)$. Anal. 
Calcd. for $\mathrm{C}_{15} \mathrm{H}_{18} \mathrm{ClN}_{3} \mathrm{O}$ (291.78): C, 61.75; H, 6.22; N, 14.40. Found: C, 61.85; H, 6.25; N, 14.27.

2-(4-Chlorophenyl)-5-[(2-pyrrolidinoethyl)amino]methyl-1,3,4-oxadiazole $\quad$ (4d). From chloride 1 (1.501 g, $6.553 \mathrm{mmol})$ and 1-(2-aminoethyl)pyrrolidine (1.65 mL, $13.019 \mathrm{mmol})$ as given for 4a. Yield 94\%, mp 63.5-65 ${ }^{\circ} \mathrm{C}$. IR: $3285(\mathrm{NH}), 2929,1607(\mathrm{C}=\mathrm{N}), 1559,1488,1411$, 1130, 1092 (Ar-Cl), 1001, 841, 818, 738 cm$^{-1} .{ }^{1} \mathrm{H}$ NMR: 1.75 (m, 4H, 3",4"-H), 2.15 (br s, 1H, $\mathrm{NH}), 2.50\left(\mathrm{~m}, 4 \mathrm{H}, 2^{\prime}, 5 "-\mathrm{H}\right), 2.62,2.81\left(2 \mathrm{xt}, J=6.2 \mathrm{~Hz}, 2 \mathrm{x} 2 \mathrm{H}, \mathrm{NHCH}_{2} \mathrm{CH}_{2} \mathrm{~N}\right), 4.12(\mathrm{~s}, 2 \mathrm{H}$, $\left.\mathrm{HetCH}_{2}\right), 7.50$ (d, $\left.J=8.6 \mathrm{~Hz}, 2 \mathrm{H}, 3^{\prime}, 5^{\prime}-\mathrm{H}\right), 8.00$ (d, $\left.J=8.6 \mathrm{~Hz}, 2 \mathrm{H}, 2^{\prime}, 66^{\prime}-\mathrm{H}\right) .{ }^{13} \mathrm{C}$ NMR: 23.3 (C$\left.3^{\prime}, 4^{\prime \prime}\right), 43.8\left(\mathrm{HetCH}_{2}\right), 47.6\left(\mathrm{NHCH}_{2} \mathrm{CH}_{2} \mathrm{~N}\right), 54.0\left(\mathrm{C}-2^{\prime \prime}, 5^{\prime \prime}\right), 55.4\left(\mathrm{NHCH}_{2} \mathrm{CH}_{2} \mathrm{~N}\right), 122.5\left(\mathrm{C}-1^{\prime}\right)$, 128.3, 129.5 (C-2',6', C-3',5'), 138.1 (C-4'), 164.5, 165.8 (C-2, C-5). MS: $306\left(<1, \mathrm{M}^{+}\right.$.), 194 (1), $166(1), 139\left(5,4-\mathrm{ClC}_{6} \mathrm{H}_{4} \mathrm{CO}^{+}\right), 84\left(100,\left(\mathrm{CH}_{2}\right)_{4} \mathrm{NCH}_{2}{ }^{+}\right)$. Anal. Calcd. for $\mathrm{C}_{15} \mathrm{H}_{19} \mathrm{ClN}_{4} \mathrm{O}(306.79)$ : C, 58.72; H, 6.24; N, 18.26. Found: C, 58.79; H, 6.02; N, 17.99.

2-(4-Chlorophenyl)-5-[(2-morpholinoethyl)amino]methyl-1,3,4-oxadiazole hydrochloride (4e.2HCl). From chloride 1 (1.508 g, $6.583 \mathrm{mmol})$ and 1-(2-aminoethyl)morpholine $(1.70 \mathrm{~mL}$, $12.953 \mathrm{mmol}$ ) as given for $\mathbf{4 a}$. The oily product was treated with saturated $\mathrm{HCl}$ solution in abs. diethyl ether to give $2.226 \mathrm{~g}(99 \%)$ of hydrochloride. Mp 205-208 ${ }^{\circ} \mathrm{C}$. IR: $2953\left(\mathrm{CH}_{2}\right), 2666$, 2441, $2363\left(\mathrm{NH}^{+}\right), 1611(\mathrm{C}=\mathrm{N}), 1591,1484,1111,1095(\mathrm{Ar}-\mathrm{Cl}), 1012,732 \mathrm{~cm}^{-1} .{ }^{1} \mathrm{H} \mathrm{NMR}$ (DMSO-d 6 : 3.40, 3.75, 3.92 (br m's, 15H, 6xCH $\mathrm{XH}_{2}+\mathrm{NH}_{2}{ }^{+}+\mathrm{NH}^{+}, 4.75$ (s, 2H, HetCH$), 7.74$ (d, $J$ $\left.=8.6 \mathrm{~Hz}, 2 \mathrm{H}, 3^{\prime}, 5^{\prime}-\mathrm{H}\right), 8.09$ (d, $\left.J=8.6 \mathrm{~Hz}, 2 \mathrm{H}, 2^{\prime}, 66^{\prime}-\mathrm{H}\right) .{ }^{13} \mathrm{C}$ NMR (DMSO-d 6 : 51.5 (C-2",6"), $51.6\left(\mathrm{CH}_{2}\right.$-morpholino), 63.2 (C-3",5"), 122.0 (C-1'), 128.8, 130.0 (C-2',6', C-3',5'), 137.5 (C-4'), 160.0, 164.5 (C-2, C-5). $\mathrm{NHCH}_{2}$ and $\mathrm{HetCH}_{2}$ overlapped with the DMSO signal. Anal. Calcd. for $\mathrm{C}_{15} \mathrm{H}_{21} \mathrm{Cl}_{3} \mathrm{~N}_{4} \mathrm{O}_{2}$ (395.71): C, 45.53; H, 5.35; N, 14.16. Found: C, 45.00; H, 5.18; N, 13.79 .

2-(4-Chlorophenyl)-5-[(3-hydroxypropyl)amino]methyl-1,3,4-oxadiazole (4f). From chloride 1 (1.501 g, $6.553 \mathrm{mmol})$ and 3-hydroxypropylamine $(1.00 \mathrm{~mL}, 13.074 \mathrm{mmol})$ as given for $4 \mathbf{a}$. Yield 85\%, mp 95-96.5 ${ }^{\circ} \mathrm{C}$. IR: $3250(\mathrm{NH}), 3154(\mathrm{OH}), 2932\left(\mathrm{CH}_{2}\right), 2858,1607(\mathrm{C}=\mathrm{N}), 1485$, 1411, 1090 (Ar-Cl), 1067, 1012, 956, 913, 831, $731 \mathrm{~cm}^{-1} .{ }^{1} \mathrm{H}$ NMR: 1.80 (m, 2H, $\mathrm{NHCH}_{2} \mathrm{CH}_{2} \mathrm{CH}_{2} \mathrm{OH}$ ), 2.78 (br s, 2H, NH, OH), 2.95 (t, $J=5.9 \mathrm{~Hz}, 2 \mathrm{H}, \mathrm{NHCH}_{2} \mathrm{CH}_{2} \mathrm{CH}_{2} \mathrm{OH}$ ), $3.82\left(\mathrm{t}, J=5.9 \mathrm{~Hz}, 2 \mathrm{H}, \mathrm{NHCH}_{2} \mathrm{CH}_{2} \mathrm{CH}_{2} \mathrm{OH}\right), 4.12\left(\mathrm{~s}, 2 \mathrm{H}, \operatorname{HetCH}_{2}\right), 7.50$ (d, $J=8.6 \mathrm{~Hz}, 2 \mathrm{H}$, 3',5'-H), 8.00 (d, J=8.6 Hz, 2H, 2',6'-H). ${ }^{13} \mathrm{C} \mathrm{NMR:} 31.0\left(\mathrm{NHCH}_{2} \mathbf{C H}_{2} \mathrm{CH}_{2} \mathrm{OH}\right), 43.6\left(\mathrm{HetCH}_{2}\right)$, $48.3\left(\mathrm{NHCH}_{2} \mathrm{CH}_{2} \mathrm{CH}_{2} \mathrm{OH}\right), 62.9\left(\mathrm{NHCH}_{2} \mathrm{CH}_{2} \mathrm{CH}_{2} \mathrm{OH}\right), 122.3$ (C-1'), 128.3, 129.6 (C-2',6', C3',5'), 138.3 (C-4'), 163.5, 165.3 (C-2, C-5). MS: 267 (2, $\mathrm{M}^{+} \cdot$ ), 222 (10), 194 (22, M $\mathrm{CH}_{2} \mathrm{CH}_{2} \mathrm{OH}$ ), 139 (35, 4- $\mathrm{ClC}_{6} \mathrm{H}_{4} \mathrm{CO}^{+}$), 137 (16, 4- $\left.\mathrm{ClC}_{6} \mathrm{H}_{4} \mathrm{CN}\right), 111$ (12), 102 (5), 74 (100, ${ }^{+} \mathrm{NHCH}_{2} \mathrm{CH}_{2} \mathrm{CH}_{2} \mathrm{OH}$ ). Anal. Calcd. for $\mathrm{C}_{12} \mathrm{H}_{14} \mathrm{ClN}_{3} \mathrm{O}_{2}$ (267.71): C, 53.84; H, 5.27; N, 15.70 .

5-(tert-Butylamino)methyl-2-(4-methoxyphenyl)-1,3,4-oxadiazole (5a). From chloride 2 $(1.505 \mathrm{~g}, 6.670 \mathrm{mmol})$ and tert-butylamine $(0.70 \mathrm{~mL}, 6.661 \mathrm{mmol})$ as given for $4 \mathrm{a}$. Yield $57 \%$, mp 75-77 ${ }^{\circ} \mathrm{C}$. IR: $3316(\mathrm{NH}), 2970\left(\mathrm{CH}_{3}\right), 1616(\mathrm{C}=\mathrm{N}), 1591,1502,1426,1363,1307,1259(\mathrm{C}-$ O-C, OMe), 1185, 1085, 1025, 832, 740, $702 \mathrm{~cm}^{-1} .{ }^{1} \mathrm{H}$ NMR: 1.15 (s, 9H, tBu), 3.85 (s, 3H, MeO), 4.01 (s, 2H, HetCH $), 6.95$ (d, J = 8.9 Hz, 2H, 3',5'-H), 7.96 (d, J=8.9 Hz, 2H, 2',6'-H). ${ }^{13} \mathrm{C}$ NMR: $28.7\left(\mathrm{CMe}_{3}\right), 37.8\left(\mathrm{HetCH}_{2}\right), 50.9\left(\mathbf{C M e}_{3}\right), 55.3(\mathrm{OMe}), 114.5\left(\mathrm{C}-3^{\prime}, 5^{\prime}\right), 116.6\left(\mathrm{C}-1^{\prime}\right)$, 128.8 (C-2',6'), 162.5, 165.2, 165.8 (C-2, C-5, C-4'). MS: 261 (1, M+.), 246 (80, M - Me), 189 
(53, $\mathrm{M}-\mathrm{tBuNH}), 177$ (13), 135 (100, 4-MeOC $\left.{ }_{6} \mathrm{H}_{4} \mathrm{CO}^{+}\right), 133$ (29, 4-MeOC $\left.{ }_{6} \mathrm{H}_{4} \mathrm{CN}\right), 92$ (10), 77 (16), 70 (56, $\left.\mathrm{C}_{4} \mathrm{H}_{8} \mathrm{~N}\right)$. Anal. Calcd. for $\mathrm{C}_{14} \mathrm{H}_{19} \mathrm{~N}_{3} \mathrm{O}_{2}$ (261.32): C, 64.35; H, 7.33; N, 16.08. Found: C, 64.30; H, 7.48; N, 15.99.

5-(Benzylamino)methyl-2-(4-methoxyphenyl)-1,3,4-oxadiazole hydrochloride (5b.HCl). From chloride $2(1.508 \mathrm{~g}, 6.713 \mathrm{mmol})$ and benzylamine $(0.74 \mathrm{~mL}, 6.774 \mathrm{mmol})$ as given for $4 \mathbf{a}$. The oily product was treated with saturated $\mathrm{HCl}$ solution in abs. diethyl ether to give $1.447 \mathrm{~g}$ (65\%) of hydrochloride. Mp 141-143 ${ }^{\circ} \mathrm{C}$. IR: 2929, 2728, $2600\left(\mathrm{NH}^{+}\right), 1616(\mathrm{C}=\mathrm{N}), 1500,1450$, 1308, 1255 (C-O-C, OMe), 1176, 1024, 844, 743, $701 \mathrm{~cm}^{-1} .{ }^{1} \mathrm{H}$ NMR (DMSO-d $)$ ): 3.87 (s, 3H, OMe), 4.35 (s, 2H, $\mathrm{PhCH}_{2} \mathrm{CH}_{2}$ ), 4.59 (s, 1H, HetCH$), 7.19$ (d, J=8.9 Hz, 2H, 3',5'-H), 7.43 (m, 3H, 2",4",6"-H), 7.59 (m, 2H, 3",5"-H), 7.98 (d, J=8.9 Hz, 2H, 3',5'-H), 10.4 (br s, 2H, $\mathrm{NH}_{2}^{+}$). ${ }^{13} \mathrm{C}$ NMR (DMSO-d $)$ : 50.0, $55.6\left(\mathrm{PhCH}_{2} \mathbf{C H}_{2}, \mathrm{HetCH}_{2}\right), 115.2\left(\mathrm{C}-3^{\prime}, 5^{\prime}\right), 115.4\left(\mathrm{C}-1^{\prime}\right), 128.8(\mathrm{C}-$ 2',6' + C-3",5"), 129.3 (C-4")*, 130.6 (C-2",6"), 131.7 (C-1")*, 159.6, 162.6, 165.2 (C-2, C-5, C4'). *Interchangeable assignment. MS: 190 (60, 2-Ar-5-Me-1,3,4-oxadiazole), 135 (27, 4$\left.\mathrm{MeOC}_{6} \mathrm{H}_{4} \mathrm{CO}^{+}\right), 133$ (30, 4-MeOC $\left.\mathrm{H}_{4} \mathrm{CN}\right), 106$ (100, $\left.\mathrm{PhCH}_{2} \mathrm{NH}\right), 91$ (65, $\left.\mathrm{PhCH}_{2}\right), 77(16)$. Anal. Calcd. for $\mathrm{C}_{16} \mathrm{H}_{21} \mathrm{~N}_{3} \mathrm{O}_{2}$ (287.36): C, 66.88; H, 7.37; N, 14.62. Found: C, 67.01; H, 7.17; N, 14.39. 5-(Cyclohexylamino)methyl-2-(4-methoxyphenyl)-1,3,4-oxadiazole (5c). From chloride 2 $(1.497 \mathrm{~g}, 6.664 \mathrm{mmol})$ and cyclohexylamine $(0.77 \mathrm{~mL}, 6.731 \mathrm{mmol})$ as given for $4 \mathbf{a}$. Yield $64 \%$, mp 80-82.5 ${ }^{\circ} \mathrm{C}$. IR: $3313(\mathrm{NH}), 2926\left(\mathrm{CH}_{2}\right), 2851\left(\mathrm{CH}_{2}\right), 1616(\mathrm{C}=\mathrm{N}), 1504,1426,1309,1256$ (C-O-C, OMe), 1181, 1086, 1032, 1005, 844, $740 \mathrm{~cm}^{-1} .{ }^{1} \mathrm{H}$ NMR: 1.09-1.31 (m, 4H, cyclohexyl $\mathrm{CH}_{2}$ ), 1.62-1.98 (m, 6H, cyclohexyl $\left.\mathrm{CH}_{2}\right), 2.05$ (m, 1H, NHCH), 3.92 (s, 3H, OMe), 4.12 (s, 2H, $\left.\mathrm{HetCH}_{2}\right), 7.01$ (d, $\left.J=9.0 \mathrm{~Hz}, 2 \mathrm{H}, 3^{\prime}, 5^{\prime}-\mathrm{H}\right), 8.00$ (d, $\left.J=9.0 \mathrm{~Hz}, 2 \mathrm{H}, 2^{\prime}, 66^{\prime}-\mathrm{H}\right) .{ }^{13} \mathrm{C}$ NMR: 24.5 (C3",5"), 25.7 (C-4"), 32.9 (C-2",6"), $40.7\left(\mathrm{HetCH}_{2}\right)$, 55.3, 55.8 (C-1", MeO), 114.5 (C-3',5'), 116.5 (C-1'), 128.8 (C-2',6'), 162.5, 165.2 (C-2, C-5, C-4'). MS: 244 (2, M - $\left.\mathrm{C}_{3} \mathrm{H}_{7}\right), 224$ (32), 189 (20), 135 (100, 4- $\left.\mathrm{MeOC}_{6} \mathrm{H}_{4} \mathrm{CO}^{+}\right), 133$ (22, 4-MeOC $\left.\mathrm{H}_{4} \mathrm{CN}\right), 119$ (8), 92 (13), 77 (20). Anal. Calcd. for $\mathrm{C}_{16} \mathrm{H}_{21} \mathrm{~N}_{3} \mathrm{O}_{2}$ (287.36): C, 66.88; H, 7.37; N, 14.62. Found: $\mathrm{C}, 66.95 ; \mathrm{H}, 7.21 ; \mathrm{N}, 14.55$.

2-(4-Methoxyphenyl)-5-[(2-pyrrolidinoethyl)amino]methyl-1,3,4-oxadiazole hydrochloride (5d.2HCl). From chloride $2(1.498 \mathrm{~g}, 6.668 \mathrm{mmol})$ and 1-(2-aminoethyl)pyrrolidine $(0.85 \mathrm{~mL}$, $6.707 \mathrm{mmol}$ ) as given for $\mathbf{4 a}$. The oily product was treated with saturated $\mathrm{HCl}$ solution in abs. diethyl ether to give $1.047 \mathrm{~g}(46 \%)$ of hydrochloride. Mp 195-200 ${ }^{\circ} \mathrm{C}$. IR: $2947\left(\mathrm{CH}_{2}\right), 2924$ $\left(\mathrm{CH}_{2}\right), 2675,2600,2363\left(\mathrm{NH}^{+}\right), 1616(\mathrm{C}=\mathrm{N}), 1500,1260$ (C-O-C, OMe), 1175, 1025, 841, $741 \mathrm{~m}^{-1} .{ }^{1} \mathrm{H}$ NMR (DMSO-d $)$ ) 3.40 (br s, 3H, $\mathrm{NH}^{+}, \mathrm{NH}_{2}^{+}$), 3.65, 3.86, $3.89\left(3 \mathrm{xs}, 12 \mathrm{H}, \mathrm{CH}_{2}\right.$ ), 4.74 (s, 2H, HetCH$), 7.22$ (d, $\left.J=8.8 \mathrm{~Hz}, 2 \mathrm{H}, 33^{\prime}, 5^{\prime}-\mathrm{H}\right), 8.04$ (d, $\left.J=8.8 \mathrm{~Hz}, 2 \mathrm{H}, 2^{\prime}, 6{ }^{\prime}-\mathrm{H}\right) .{ }^{13} \mathrm{C}$ NMR (DMSO-d $)$ : 22.6 (C-3",4"), $42.7\left(\mathrm{HetCH}_{2}\right), 49.2,55.6\left(\mathrm{NCH}_{2} \mathrm{CH}_{2} \mathrm{~N}\right), 53.2\left(\mathrm{C}-2^{\prime \prime}, 5 "\right)$, 115.2 (C-3',5'), 115.4 (C-1'), 128.9 (C-2',6'), 159.4, 162.7, 165.2 (C-2, C-5, C-4'). MS: 302 (1, $\mathrm{M}$ - of free base), 190(1), $135\left(15,4-\mathrm{MeOC}_{6} \mathrm{H}_{4} \mathrm{CO}^{+}\right), 111(3), 84\left(100,\left(\mathrm{CH}_{2}\right){ }_{4} \mathrm{NCH}_{2}^{+}\right), 78(38)$. Anal. Calcd. for $\mathrm{C}_{16} \mathrm{H}_{24} \mathrm{Cl}_{2} \mathrm{~N}_{4} \mathrm{O}_{2}$ (375.29): C, 51.21; H, 6.45; N, 14.93. Found: C, 50.56; H, 6.41; $\mathrm{N}, 14.58$.

2-(4-Chlorophenyl)-5-[(2-pyridyl)thio]methyl-1,3,4-oxadiazole (6a). From chloride 1 $(1.506 \mathrm{~g}, 6.575 \mathrm{mmol})$ and 2-mercaptopyridine $(0.734 \mathrm{~g}, 6.603 \mathrm{mmol})$ as given for 4a. Yield 86\%, mp 89-90.5 ${ }^{\circ} \mathrm{C}$. IR: 2914, $1606(\mathrm{C}=\mathrm{N}), 1578,1558,1483,1456,1412,1256,1130,1092$ 
(Ar-Cl), 1008, 834, 756, $733 \mathrm{~cm}^{-1} .{ }^{1} \mathrm{H}$ NMR (DMSO-d $)$ ): 4.80 (s, 2H, HetCH $), 7.20$ (dd, J = 7.3, $\left.5.2 \mathrm{~Hz}, 1 \mathrm{H}, 5^{\prime}-\mathrm{H}\right), 7.45$ (d, J=8.1 Hz, 1H, 3"-H), 7.70 (m, 3H, 3',5'-H), 7.94 (d, J = 8.6 Hz, 2H, $\left.2^{\prime}, 6^{\prime}-\mathrm{H}\right), 8.43\left(\mathrm{~d}, J=5.8 \mathrm{~Hz}, 1 \mathrm{H}, 66^{\prime \prime}-\mathrm{H}\right) .{ }^{13} \mathrm{C}$ NMR (DMSO-d 6$): 22.7\left(\mathrm{HetCH}_{2}\right), 120.9,122.2(\mathrm{C}-$ 1', C-3", C-5"), 128.4, 129.9 (C-2',6', C-3',5), 137.0 (C-4'), 137.4 (C-4"), 149.8 (C-6"), 155.8 (C2"), 163.8, 165.0 (C-2, C-5). MS: 303/305 (63/21, M+., $\left.\mathrm{Cl}_{35} / \mathrm{Cl}_{37}\right), 270$ (6), 230 (8), 194 (6), 164 (47), $139\left(77,4-\mathrm{ClC}_{6} \mathrm{H}_{4} \mathrm{CO}^{+}\right), 137\left(43,4-\mathrm{ClC}_{6} \mathrm{H}_{4} \mathrm{CN}\right), 124\left(75,2-\mathrm{pySCH}_{2}{ }^{+}\right), 123$ (100), 111 (83, 2-pySH), 78 (80, py ${ }^{+}$). Anal. Calcd. for $\mathrm{C}_{14} \mathrm{H}_{10} \mathrm{ClN}_{3} \mathrm{OS}$ (303.76): C, 55.36; H, 3.32; N, 13.83 . Found: C, 55.35; H, 3.11; N, 14.02 .

5-[(2-Benzimidazolyl)thio]methyl-2-(4-chlorophenyl)-1,3,4-oxadiazole (6b). From chloride 1 (1.501 g, $6.553 \mathrm{mmol})$ and 2-mercaptobenzimidazol $(0.985 \mathrm{~g}, 6.558 \mathrm{mmol})$ as given for 4a. Yield 86\%, mp 205-206 ${ }^{\circ} \mathrm{C}$. IR: 3073, 2985, 2881, 2811, $1609(\mathrm{C}=\mathrm{N}), 1566,1483,1403,1356$, 1280, 1092 (Ar-Cl), 1011, 834, 752, $724 \mathrm{~cm}^{-1} .{ }^{1} \mathrm{H}$ NMR (DMSO-d 6 ): 4.45 (s, 2H, HetCH $), 7.18$ (m, 2H, 5",6"-H), 7.50 (br s, 2H, 4",7"-H), 7.62 (d, J=8.3 Hz, 2H, 3',5'-H), 7.90 (d, J=8.3 Hz, 2H, 2',6'-H), 12.80 (s, 1H, NH). ${ }^{13} \mathrm{C}$ NMR: $25.3\left(\mathrm{HetCH}_{2}\right), 122.2\left(\mathrm{C}-1^{\prime}\right), 128.4,129.8$ (C-2',6', C3',5'), 137.0 (C-4'), 147.7 (C-2"), 163.9, 164.5 (C-2, C-5). C-4", C-3a", C-5", C-6", C-7", C-7a" carbon signals appeared as highly broadened singlets at $\delta \sim 111,117.5,122 \mathrm{ppm}$ due to the exchange of hydrogen between the two nitrogens. MS: 342, $344\left(43 / 15, \mathrm{Cl}_{35} / \mathrm{C}_{377}\right), 194$ (26), 163 (38, benzimidazolyl- $\mathrm{SCH}_{2}^{+}$), 162 (80), 150 (90, 2-HS-benzimidazole), 139 (100, 4- $\mathrm{ClC}_{6} \mathrm{H}_{4} \mathrm{CO}^{+}$), 123 (43), 111 (43, 4- $\mathrm{ClC}_{6} \mathrm{H}_{4}{ }^{+}$), 90 (15), 75(38). Anal. Calcd. for $\mathrm{C}_{16} \mathrm{H}_{11} \mathrm{ClN}_{4} \mathrm{OS}$ (342.80): C, 56.06; H, 3.23; N, 16.34. Found: C, 55.97; H, 3.54; N, 16.20.

5-[(2-Benzoxazolyl)thio]methyl-2-(4-chlorophenyl)-1,3,4-oxadiazole (6c). From chloride 1 $(1.515 \mathrm{~g}, 6.614 \mathrm{mmol})$ and 2-mercaptobenzoxazol (0.999 g, $6.608 \mathrm{mmol})$ as given for 4a. Yield 84\%, mp 105-106 ${ }^{\circ} \mathrm{C}$. IR: 3084, $1606 \mathrm{w}(\mathrm{C}=\mathrm{N}), 1586,1502,1486,1453,1403,1238,1224$, 1140, 1095 (Ar-Cl), 1009, 850, 754, 744, $731 \mathrm{~cm}^{-1} .{ }^{1} \mathrm{H}$ NMR (DMSO-d $)$ : 4.81 (s, 2H, HetCH $)$, 7.32 (m, 2H, 5",6"-H), 7.46 (d, J = 8.5 Hz, 2H, 3',5'-H), 7.48, 7.65 (2xm, 2x1H, 4",7"-H), 7.92 (d, $\left.J=8.5 \mathrm{~Hz}, 2 \mathrm{H}, 2^{\prime}, 6^{\prime}-\mathrm{H}\right) .{ }^{13} \mathrm{C}$ NMR (DMSO-d $): 25.6\left(\mathrm{HetCH}_{2}\right), 110.2$ (C-7"), 119.0 (C-4"), 122.0 (C-1'), 124.6, 124.8 (C-5",C-6"), 128.4, 129.6 (C-2',6', C-3',5'), 138.5 (C-4'), 141.8 (C3a"), 152.5 (C-7a"), 162.1, 162.9, 165.1 (C-2, C-5, C-2"). MS: 343/345 (35/12, $\left.\mathrm{M}^{+} \cdot \mathrm{Cl}_{35} / \mathrm{Cl}_{37}\right)$, 270 (3), 193 (25), 151 (15, 2-HS-benzoxazole), 139 (100, 4- $\left.\mathrm{ClC}_{6} \mathrm{H}_{4} \mathrm{CO}^{+}\right), 123$ (22), 111 (30, 4$\mathrm{ClC}_{6} \mathrm{H}_{4}{ }^{+}$), 91 (9), 75(15). Anal. Calcd. for $\mathrm{C}_{16} \mathrm{H}_{10} \mathrm{ClN}_{3} \mathrm{O}_{2} \mathrm{~S}$ (343.78): C, 55.90; H, 2.93; N, 12.22. Found: C, 56.12; H, 2.77; N, 12.21 .

2-(4-Chlorophenyl)-5-[(1,2,4-triazol-3-yl)thio]methyl-1,3,4-oxadiazole (6d). From chloride 1 (1.502 g, $6.557 \mathrm{mmol})$ and 3-mercapto-1,2,4-triazole $(1.327 \mathrm{~g}, 13.122 \mathrm{mmol})$ as given for 4a. Yield 78\%, mp 138.5-139 ${ }^{\circ} \mathrm{C}$. IR: $3249(\mathrm{NH}), 3137,1606(\mathrm{C}=\mathrm{N}), 1565,1484,1460,1410,1274$, 1232, 1092 (Ar-Cl), 1012, 845, $729 \mathrm{~cm}^{-1} .{ }^{1} \mathrm{H}$ NMR (DMSO-d 6 ): 4.69 (s, 2H, HetCH $), 7.69$ (d, J $\left.=8.6 \mathrm{~Hz}, 2 \mathrm{H}, 3^{\prime}, 5^{\prime}-\mathrm{H}\right), 7.98\left(\mathrm{~d}, J=8.6 \mathrm{~Hz}, 2 \mathrm{H}, 3^{\prime}, 5^{\prime}-\mathrm{H}\right), 8.60(\mathrm{~s}, 1 \mathrm{H}$, triazole-H), $14.20(\mathrm{br} \mathrm{s}, 1 \mathrm{H}$, $\mathrm{NH}) .{ }^{13} \mathrm{C}$ NMR (DMSO-d 6 ): $25.4\left(\mathrm{HetCH}_{2}\right), 122.2$ (C-1'), 128.4, 129.9 (C-2',6', C-3',5'), 137.0 (C-4'), 145.6 br (triazole CH), 163.8, 164.7 (C-2, C-5). MS: 293/295 (25/8, M+., $\left.\mathrm{Cl}_{35} / \mathrm{Cl}_{37}\right), 193$ (8), 139 (100, 4- $\left.\mathrm{ClC}_{6} \mathrm{H}_{4} \mathrm{CO}^{+}\right), 113$ (61), 111(61, 4- $\left.\mathrm{ClC}_{6} \mathrm{H}_{4}{ }^{+}\right)$, 75(40). Anal. Calcd. for $\mathrm{C}_{11} \mathrm{H}_{8} \mathrm{ClN}_{5} \mathrm{OS}$ (293.73): C, 44.98; H, 2.75; N, 23.84. Found: C, 45.21; H, 2.88; N, 23.44. 
2-(4-Methoxyphenyl)-5-[(2-pyridyl)thio]methyl-1,3,4-oxadiazole (7a). From chloride 2 $(1.531 \mathrm{~g}, 6.815 \mathrm{mmol})$ and 2-mercaptopyridine $(0.761 \mathrm{~g}, 6.845 \mathrm{mmol})$ as given for 4a. Yield 84\%, mp 49.5-51 ${ }^{\circ} \mathrm{C}$. IR: 2999, $1615(\mathrm{C}=\mathrm{N}), 1591,1557,1500,1458,1425,1261$ (C-O-C, OMe), 1130, 1086, 1029, 838, $756 \mathrm{~cm}^{-1} .{ }^{1} \mathrm{H}$ NMR (DMSO-d $): 3.85$ (s, 3H, OMe), 4.73 (s, 2H, $\left.\mathrm{HetCH}_{2}\right), 6.96\left(\mathrm{~d}, J=9.0 \mathrm{~Hz}, 2 \mathrm{H}, 3^{\prime}, 5^{\prime}-\mathrm{H}\right), 7.06$ (ddd, $\left.J=7.1,4.9,1.0 \mathrm{~Hz}, 1 \mathrm{H}, 5^{\prime \prime}-\mathrm{H}\right), 7.26$ (dd, $J$ $\left.=8.2,1.1 \mathrm{~Hz}, 1 \mathrm{H}, 3^{\prime \prime}-\mathrm{H}\right), 7.54$ (ddd, $\left.J=8.2,7.1,1.9 \mathrm{~Hz}, 1 \mathrm{H}, 4^{\prime \prime}-\mathrm{H}\right), 7.91$ (d, $J=9.0 \mathrm{~Hz}, 2 \mathrm{H}, 2^{\prime}, 6^{\prime}-$ $\mathrm{H}), 8.48(\mathrm{dd}, J=4.9,1.9 \mathrm{~Hz}, 1 \mathrm{H}, 6 "-\mathrm{H}) .{ }^{13} \mathrm{C} \mathrm{NMR}\left(\mathrm{DMSO}-\mathrm{d}_{6}\right): 23.2\left(\mathrm{HetCH}_{2}\right), 55.3(\mathrm{OMe})$, 114.5 (C-3',5'), 116.4 (C-1'), 120.4, 122.4 (C-3',5"), 128.7 (C-2',6'), 136.5 (C-4"), 149.7 (C-6"), 156.0 (C-2"), 162.5, 163.8, 165.4 (C-2, C-5, C-4'). MS: 299 (56, M+), 226 (10), 164 (25), 149 (10), 135 (100, 4- $\left.\mathrm{MeOC}_{6} \mathrm{H}_{4} \mathrm{CO}^{+}\right), 124$ (43, 2-pySCH$\left.{ }^{+}\right), 123$ (50), 111 (19, 2-pySH), 92 (26), 78 $\left(50, \mathrm{py}^{+}\right)$. Anal. Calcd. for $\mathrm{C}_{15} \mathrm{H}_{13} \mathrm{~N}_{3} \mathrm{O}_{2} \mathrm{~S}$ (299.34): C, 60.19; H, 4.38; N, 14.04. Found: C, 59.89; H, 4.29; N, 14.05 .

5-[(2-Benzimidazolyl)thio]methyl-2-(4-methoxyphenyl)-1,3,4-oxadiazole (7b). From chloride $2(1.510 \mathrm{~g}, 6.722 \mathrm{mmol})$ and 2-mercaptobenzimidazole $(1.011 \mathrm{~g}, 6.731 \mathrm{mmol})$ as given for $4 \mathbf{a}$. Yield 89\%, mp 199-203 ${ }^{\circ} \mathrm{C}$. IR: $1616(\mathrm{C}=\mathrm{N}), 1558,1499,1410,1307,1261$ (C-O-C, OMe), 1177, 1017, 1007, 843, 824, $735 \mathrm{~cm}^{-1} .{ }^{1} \mathrm{H}$ NMR (DMSO-d $)$ ) 3.83 (s, 3H, OMe), 4.90 (s, 2H, $\left.\mathrm{HetCH}_{2}\right), 7.08$ (d, J = 8.9 Hz, 1H, 3',5'-H), 7.15 (m, 2H, 5",6"-H), 7.43, 7.55 (2xbr s, 2x1H, 4",7"-H), 7.81 (d, J = 8.9 Hz, 2H, 2',5'-H), 12.80 (s, 1H, NH). ${ }^{13} \mathrm{C}$ NMR (DMSO-d 6 ): 25.2 $\left(\mathrm{HetCH}_{2}\right), 55.4(\mathrm{MeO}), 110.8^{*}$ (C-7"), 114.9 (C-3',5'), 115.7 (C-1'), 117.9* (C-4"), 121.6*, 122.2* (C-5",6"), 128.4 (C-2',6'), 135.8*, 143.8* (C-3a",C-7a"), 147.7 (C-2"), 162.3, 163.5, 164.6 (C-2, C-5, C-4'). Signals denoted with * slightly broadened due to the exchange of hydrogen between the two nitrogens. MS: $338\left(20, \mathrm{M}^{+}\right), 190$ (8), 189 (5), 163 (12, benzimidazolyl- $\mathrm{SCH}_{2}{ }^{+}$), 162 (20), 150 (19, 2-SH-benzimidazole), 135 (100, 4- $\mathrm{MeOC}_{6} \mathrm{H}_{4} \mathrm{CO}^{+}$), 107(8, 4- $\mathrm{MeOC}_{6} \mathrm{H}_{4}^{+}$), 92 (20), 77 (22). Anal. Calcd. for $\mathrm{C}_{17} \mathrm{H}_{14} \mathrm{~N}_{4} \mathrm{O}_{2} \mathrm{~S}$ (338.38): C, 60.34; $\mathrm{H}$, 4.17; N, 16.56. Found: C, 60.66; H, 3.99; N, 16.47 .

5-[(2-Benzothiazolyl)thio]methyl-2-(4-methoxyphenyl)-1,3,4-oxadiazole (7e). From chloride $2(1.859 \mathrm{~g}, 8.275 \mathrm{mmol})$ and 2-mercaptobenzothiazole $(1.390 \mathrm{~g}, 8.311 \mathrm{mmol})$ as given for $4 \mathbf{a}$. Yield 76\%, mp 88-92 ${ }^{\circ} \mathrm{C}$. IR: $1613(\mathrm{C}=\mathrm{N}), 1585,1500,1463,1427,1307,1225$ (C-O-C, OMe), 1175, 1018, 997, 846, $755 \mathrm{~cm}^{-1} .{ }^{1} \mathrm{H}$ NMR: 3.86 (s, 3H, OMe), 4.89 (s, 2H, HetCH$), 6.96$ (d, J= $\left.9.0 \mathrm{~Hz}, 2 \mathrm{H}, 3^{\prime}, 5^{\prime}-\mathrm{H}\right), 7.34,7.46$ (2xm, 2x1H, 5",6"-H), 7.79 (dd, J = 7.6, $\left.1.5 \mathrm{~Hz}, 1 \mathrm{H}, 8^{\prime \prime}-\mathrm{H}\right), 7.90$ (m, 3H, 2',6'-H, 4"-H). ${ }^{13} \mathrm{C}$ NMR: $26.4\left(\mathrm{HetCH}_{2}\right), 55.3(\mathrm{OMe}), 114.5\left(\mathrm{C}-3^{\prime}, 5^{\prime}\right), 116.1$ (C-1'), 121.2, 122.1, 124.9, 126.4 (C-4", C-5", C-6", C-7"), 128.8 (C-2',6'), 135.8 (C-7a"), 153.0 (C-2"), 162.4, 162.7, 163.6, 165.7 (C-2, C-5, C-4', C-3a"). MS: 355 (12, M+), 179 (8), 167 (15, 2-HSbenzothiazole), 135 (100, 4- $\left.\mathrm{MeOC}_{6} \mathrm{H}_{4} \mathrm{CO}^{+}\right), 108$ (10), 92 (10), 77 (12). Anal. Calcd. for $\mathrm{C}_{17} \mathrm{H}_{13} \mathrm{~N}_{3} \mathrm{O}_{2} \mathrm{~S}_{2}$ (355.42): C, 57.45; H, 3.69; N, 11.82. Found: C, 54.55; H, 3.34; N, 11.90 .

5-[(2-Benzimidazolyl)sulfinyl]methyl-2-(4-chlorophenyl)-1,3,4-oxadiazole (8b). Typical procedure. $21 \mathrm{~mL}$ of $0.074 \mathrm{M}$ dimethyldioxirane solution in acetone (ca. 2.1 equiv.) was added to a solution of $\mathbf{6 b}(0.256 \mathrm{~g}, 0.747 \mathrm{mmol})$ in acetone $(5 \mathrm{~mL})$. The mixture was stirred for 30 minutes at room temperature and monitored by TLC $(\mathrm{MeOH}-\mathrm{PhMe}=5: 1, \mathrm{v} / \mathrm{v})$. After completion $(30 \mathrm{~min}$ ), the solvent was removed under reduced pressure to obtain $239 \mathrm{mg}$ 
yellowish crystalline solid which proved to be a $92 / 8$ mixture of sulfoxide $\mathbf{8 b}$ and sulfone $10 \mathrm{~b}$ by ${ }^{1} \mathrm{H}$ NMR analysis. The crude product was purified by column chromatography (hexane- $\mathrm{Me}_{2} \mathrm{CO}=$ 1:1, v/v) to give $198 \mathrm{mg}(74 \%)$ of pure 8b. mp $187-190{ }^{\circ} \mathrm{C}$. IR: $3220(\mathrm{NH}), 2928,1606(\mathrm{C}=\mathrm{N})$, 1554, 1484, 1400, 1270, $1094 \mathrm{Ar}-\mathrm{Cl}), 1058,1050(\mathrm{~S}=\mathrm{O}), 1012,832,746,730 \mathrm{~cm}^{-1} .{ }^{1} \mathrm{H}$ NMR (DMSO-d $\left.\mathrm{d}_{6}\right): 4.94\left(\mathrm{~d}, J=14.1 \mathrm{~Hz}, 1 \mathrm{H}\right.$, one of $\left.\mathrm{HetCH}_{2}\right), 5.26(\mathrm{~d}, J=14.1 \mathrm{~Hz}, 1 \mathrm{H}$, the other $\mathrm{HetCH}_{2}$ ), 7.34 (m, 2H, 5",6"--H), 7.48 (A2B2, 4H, 2',3',5',6'-H), 7.65 (br s, 2H, 4",7"-H). MS: 358 (70, $\left.\mathrm{M}^{+}\right), 284$ (93), 282 (42), 240 (82, M - benzimidazole), 210 (100), 208 (58), 181 (19), 150 (36, 2-HS-benzimidazole), $139\left(69,4-\mathrm{ClC}_{6} \mathrm{H}_{4} \mathrm{CO}^{+}\right), 111\left(36,4-\mathrm{ClC}_{6} \mathrm{H}_{4}{ }^{+}\right)$. Anal. Calcd. for $\mathrm{C}_{16} \mathrm{H}_{11} \mathrm{ClN}_{4} \mathrm{O}_{2} \mathrm{~S}$ (358.80): C, 53.56; H, 3.09; N, 15.61. Found: C, 53.64; H, 2.99; N, 15.82 .

5-[(2-Benzimidazolyl)sulfinyl]methyl-2-(4-methoxyphenyl)-1,3,4-oxadiazole (9b). From sulfide $7 \mathbf{b}(0.251 \mathrm{~g}, 0.742 \mathrm{mmol})$ with $61 \mathrm{~mL} 0.049 \mathrm{M}$ dimethyldioxirane solution (ca. 4.0 equiv.) according to the procedure given for $\mathbf{8 b} 0.247 \mathrm{~g}$ crude product $\left(\mathbf{9 b} / \mathbf{1 1} \mathbf{b}=9 / 1,{ }^{1} \mathrm{H}\right.$ NMR) was obtained which yielded $0.197 \mathrm{mg}(75 \%)$ pure $\mathbf{9 b}$ after column chromatography. mp 155158.3 ${ }^{\circ} \mathrm{C}$. IR: 2928, $1612(\mathrm{C}=\mathrm{N}), 1498,1428,1402,1306,1260$ (C-O-C, OMe), 1174, 1064 $(\mathrm{S}=\mathrm{O}), 1028,838,740 \mathrm{~cm}^{-1} .{ }^{1} \mathrm{H}$ NMR (DMSO-d $)$ ) 3.80 (s, 3H, MeO), 4.91 (d, $J=13.9 \mathrm{~Hz}, 1 \mathrm{H}$, one of $\left.\mathrm{HetCH}_{2}\right), 5.23\left(\mathrm{~d}, J=13.9 \mathrm{~Hz}, 1 \mathrm{H}\right.$, the other HetCH $\left.\mathbf{H}_{2}\right), 6.94\left(\mathrm{~d}, J=8.4 \mathrm{~Hz}, 2 \mathrm{H}, 3^{\prime}, 5^{\prime}-\mathrm{H}\right)$, 7.34 (m, 2H, 5",6"-H), 7.41 (d, J = 8.4 Hz, 2H, 2',6'-H), 7.64 (br s, 2H, 4",7"-H). MS: 355 (5, M + 1), $354\left(2, \mathrm{M}^{+}\right.$.), 338 (1, M - O), 336 (2, M - $\mathrm{H}_{2} \mathrm{O}$ ), 321 (3, M - $\left.\mathrm{H}_{2} \mathrm{O}-\mathrm{Me}\right), 237$ (8), 190 (30), 150 (37, 2-HS-benzimidazole), 135 (100, 4-MeOC $\left.6 \mathrm{H}_{4} \mathrm{CO}^{+}\right), 133$ (25, 4- $\left.\mathrm{MeOC}_{6} \mathrm{H}_{4} \mathrm{CN}\right), 73$ (35). Anal. Calcd. for $\mathrm{C}_{17} \mathrm{H}_{14} \mathrm{~N}_{4} \mathrm{O}_{3} \mathrm{~S}$ (354.38): C, 57.62; H, 3.98; N, 15.81. Found: C, 57.66; H, 4.15; $\mathrm{N}, 15.97$.

5-[(2-Benzimidazolyl)sulfonyl]methyl-2-(4-chlorophenyl)-1,3,4-oxadiazole $\quad$ (10b). From sulfide $6 \mathbf{b}(0.252 \mathrm{~g}, 0.732 \mathrm{mmol})$ with $58 \mathrm{~mL} 0.065 \mathrm{M}$ dimethyldioxirane solution (ca. 5.2 equiv.) according to the procedure given for $\mathbf{8 b}$ after removal of the solvent $0.247 \mathrm{~g} \mathrm{(90 \% )} \mathrm{pure}$ 10b sulfone was obtained. mp 190-193. ${ }^{\circ} \mathrm{C}$. IR: $3088(\mathrm{NH}), 1608(\mathrm{C}=\mathrm{N}), 1482,1412,1356$, $1344\left(\mathrm{SO}_{2}\right), 1200,1160,1142\left(\mathrm{SO}_{2}\right), 1094,1012,836,806,746 \mathrm{~cm}^{-1} .{ }^{1} \mathrm{H}$ NMR (DMSO-d 6 ): 5.68 (s, 2H, HetCH $), 7.46$ (br s, 2H, 5",6"-H), 7.58 (d, $\left.J=8.4 \mathrm{~Hz}, 2 \mathrm{H}, 3^{\prime}, 5^{\prime}-\mathrm{H}\right), 7.66$ (d, $J=8.4 \mathrm{~Hz}$, 2H, 2',6'-H), 7.84 (br s, 2H, 4",7"-H). MS: 310, 312 (48/15, M - SO2, Cl35/Cl37), 268 (44), 194 (16), 173 (56), $139\left(100,4-\mathrm{ClC}_{6} \mathrm{H}_{4} \mathrm{CO}^{+}\right), 111\left(56,4-\mathrm{ClC}_{6} \mathrm{H}_{4}{ }^{+}\right), 90$ (35). Anal. Calcd. for $\mathrm{C}_{16} \mathrm{H}_{11} \mathrm{ClN}_{4} \mathrm{O}_{3} \mathrm{~S}$ (374.80): C, 51.27; H, 2.96; N, 14.95. Found: $\mathrm{C}, 51.02 ; \mathrm{H}, 3.13 ; \mathrm{N}, 15.11$.

5-[(2-Benzimidazolyl)sulfonyl]methyl-2-(4-methoxyphenyl)-1,3,4-oxadiazole (11b). From sulfide $7 \mathbf{b}(0.252 \mathrm{~g}, 0.745 \mathrm{mmol})$ with $76 \mathrm{~mL} 0.079 \mathrm{M}$ dimethyldioxirane solution (ca. 8.1 equiv.) according to the procedure given for $\mathbf{8 b}$ after removal of the solvent $0.250 \mathrm{~g}(91 \%)$ pure 11b sulfone was obtained. mp 192-194 ${ }^{\circ} \mathrm{C}$. IR: $3082(\mathrm{NH}), 1614(\mathrm{C}=\mathrm{N}), 1498,1340\left(\mathrm{SO}_{2}\right), 1262$ (C-O-C), 1186, 1176, $1144\left(\mathrm{SO}_{2}\right), 1020,1006,840,806,742 \mathrm{~cm}^{-1} .{ }^{1} \mathrm{H}$ NMR (DMSO-d $)$ ): 3.82 (s, $3 \mathrm{H}, \mathrm{OMe}), 5.64$ (s, 2H, HetCH ), 7.02 (d, J = 9.1 Hz, 2H, 3',5'-H), 7.45 (m, 2H, 5",6"-H), 7.58 (d, $\left.J=9.1 \mathrm{~Hz}, 2 \mathrm{H}, 2^{\prime}, 6^{\prime}-\mathrm{H}\right), 7.74$ (br s, 2H, 4",7"-H). MS: 306 (31, M - SO2), 264 (24), 221 (45), 189 (15), 173 (17), 157 (20), 135 (100, 4- $\left.\mathrm{MeOC}_{2} \mathrm{H}_{6} \mathrm{CO}^{+}\right), 118$ (9), 81(40). Anal. Calcd. for $\mathrm{C}_{17} \mathrm{H}_{14} \mathrm{~N}_{4} \mathrm{O}_{4} \mathrm{~S}$ (370.38): C, 55.13; H, 3.81; N, 15.13. Found: C, 55.42; H, 3.85; N, 15.02. 


\section{Acknowledgements}

The financial support of Hungarian Scientific Research Found (OTKA 22290) is highly appreciated.

\section{References}

1. Hill, J. 1,3,4-Oxadiazoles In Comprehensive Heterocyclic Chemistry; Katritzky, A. R.; Rees, C. W. Pergamon: Oxford-New York-Toronto-Sydney-Paris-Frankfurt, 1984; Vol. 6, 427.

2. Brown, P.; Best, D. J.; Broom, N. J. P.; Cassels, R.; O'Hanlon, P. J.; Mitchell, T. J.; Osborne, N. F.; Wilson, J. M. J. Med. Chem. 1997, 40, 2563.

3. Girges, M. M. Arzneim.-Forsch./Drug Res. 1994, 44, 490.

4. Singh, H.; Yadav, L. D. S.; Chaudhary, J. P. Acta Chim. Hung. 1985, 118, 11.

5. Angelini, I.; Angelini, L.; Sparaco, F. Brit. Pat. 1.161 .801 (1966/1969); Chem. Abstr. 1969, $71,112937$.

6. Palazzo, G.; Silvestrini, B. US Pat. 3.502.888 (1966/1970); Chem. Abstr. 1970, 72, 132714.

7. Rieber, N.; Böhm, H. J. Heterocycl. Chem. 1981, 18, 1.

8. Baiocchi, L.; Picconi, G.; Palazzo, G. J. Heterocycl. Chem. 1979, 16, 1479.

9. Pohl, M.; Thieme, M.; Jones, P. G.; Liebscher, J. Liebigs Ann. Chem. 1995, 1539.

10. Pätzel, M.; Bohrisch, J.; Liebscher, J. Liebigs Ann. Chem. 1991, 975.

11. Angelini, I.; Angelini, L.; Sparaco, F. Brit. Pat. 1.161 .802 (1966/1969); Chem. Abstr. 1969, $71,112936$.

12. Huisgen, R.; Sauer, J.; Sturm, H. J.; Markgraf, J. H. Chem. Ber. 1960, 93, 2106.

13. Faber, K.; Kappe, T. J. Heterocycl. Chem. 1984, 21, 1881.

14. Jilale, A.; Nechitaïlo, P.; Decroix, B.; Végh, D. J. Heterocycl. Chem. 1993, 30, 881.

15. Vakula, T. R.; Srinivasan, V. R. Indian J. Chem. 1973, 11, 732

16. (a) Adam, W.; Curci, R.; Edwards, J. O. Acc. Chem. Res. 1989, 22, 205. (b) Murray, R. W. Chem. Rev. 1989, 89, 1187. (c) Adam, W.; Hadjiarapoglou, L. Top. Curr. Chem. 1993, 164, 45. (d) Curci, R.; Dinoi, A.; Rubino, M. F. Pure Appl. Chem. 1995, 67, 811. (e) Adam, W.; Smerz, K. Bull. Soc. Chim. Belg. 1996, 105, 581.

17. Patonay, T.; Adam, W.; Lévai, A.; Kövér, P.; Németh, M.; Peters, E.-M.; Peters, K. J. Org. Chem. 2001, 66, 2275.

18. Adam, W.; Bialas, J.; Hadjiarapoglou, L. Chem. Ber. 1991, 124, 2377.

19. Dost, J.; Heschel, M.; Stein, J. J. Prakt. Chem. 1985, 327, 109.

20. Hogale, M. B.; Shelar, A. R.; Kachare, D. S.; Salunkhe, V. K. J. Indian Chem. Soc. 1987, 64, 314. 